\title{
Estado nutricional e desfechos clínicos em pacientes críticos internados em hospital universitário
}

\author{
Nutritional status and clinical outcomes in critically ill patients admitted to a university hospital
}

\section{DOI: $10.37111 /$ braspeni.2019344009}

Edcleide Oliveira dos Santos Olinto

Gina Araújo Martins Feitosa²

Izaura Odir Lima Gomes da Costa ${ }^{3}$

Janine Maciel Barbosa ${ }^{4}$

Ericka Vilar Bôtto Targino 5

Paloma Egídio Andrade de Sousa ${ }^{6}$

Camila Melo de Araújo ${ }^{6}$

\begin{abstract}
RESUMO
Introdução: Há forte relação da desnutrição com o aumento do tempo de internação e morbimortalidade. Estudos demonstram que os pacientes desnutridos podem ter até vinte vezes mais complicações que os eutróficos. Em pacientes críticos, há tendência ao catabolismo, resultando na perda de massa magra que, quando atinge $40 \%$, geralmente é letal. Método: Pesquisa quantitativa, descritiva, realizada com adultos, de ambos os sexos, admitidos na unidade de terapia intensiva de um hospital universitário, de março a dezembro de 2018. Foram coletadas das fichas de acompanhamento nutricional, as variáveis gênero, idade (I), período de internamento na UTI, data de alta ou óbito, risco nutricional através da triagem, além do peso $(P)$, altura $(A)$ e circunferência braquial (CB). Para a triagem foi utilizado o Nutric escore, para a avaliação nutricional foram utilizados os indicadores índice de massa corporal (IMC) e CB e as classificações recomendadas pela Organização Mundial de Saúde (2004) e Blackburn e Thornton (1979). Após a coleta, os dados foram analisados no Statistical Package for the Social Sciences (SPSS) 13.0 e para a associação das variáveis foi utilizado o teste de Qui-Quadrado, considerando diferença estatística quando o valor de $p<0,05$. Resultados: Amostra foi composta por 116 pacientes a maioria do gênero feminino $(53,4 \%)$, cuja mediana de idade foi de 46 anos (IQ $31-53$ ). Em relação à frequência do risco nutricional, a maioria dos pacientes $(61,5 \%)$ tinha escore baixo. Houve uma frequência importante de desnutrição, segundo o indicador $C B(73 \%)$, embora pelo IMC $(43,5 \%)$ tenha demonstrado eutrofia. Ainda que a maioria dos pacientes tenha apresentado risco nutricional baixo, entre aqueles que apresentaram risco nutricional alto $(38,5 \%)$ houve tendência maior à mortalidade, no entanto, sem confirmação estatística $(p>0,05)$. Houve, também, tendência $(p>0,05)$ de associação de óbito com a desnutrição, embora sem significância estatística. Conclusão: Os pacientes com risco nutricional e/ou desnutrição parecem estar vulneráveis a piores desfechos clínicos.
\end{abstract}

\section{ABSTRACT}

Introduction: There is a strong relationship between malnutrition and increased length of hospitalization and morbidity and mortality. Studies have shown that malnourished patients can have up to twenty times more complications than eutrophic ones. In critically ill patients, there is a tendency to catabolism, resulting in the loss of lean body mass, which when it reaches $40 \%$ is usually lethal. Methods: A quantitative, descriptive study was conducted on adults from both genders, admitted to the intensive care unit (ICU) of a university hospital, from March to December 2018. The following variables were collected from the evaluation and nutritional records: length of hospitalization in the ICU, date of discharge or death, nutritional risk through specific screening, height, weight and arm circumference (AC). For the screening, the Nutric score was used. For the nutritional evaluation, the body mass index (BMI) and $\mathrm{AC}$ indicators and the classifications recommended by the World Health Organization (2004) and Blackburn and Thornton (1979) were used. After collecting the data, they were analyzed in the Statistical Package for Social Sciences (SPSS) 13.0 and for the association of the variables the Chi-square test was used, considering statistical difference when the $p$ value $<0.05$. Results: The sample consisted of 116 patients, mostly female $(53.4 \%)$ whose median age was 46 years (interquartile range IQR 31-53). Regarding the frequency of nutritional risk, most patients $(61.5 \%$ ) had a low score. There was an important frequency of malnutrition, according to the AC indicator (73\%), although BMI (43.5\%) showed eutrophy. Even though most patients had low nutritional risk, those with high nutritional risk (38.5\%) had a higher tendency to mortality, however, not statistically confirmed $(p>0.05)$. There was also a tendency of association between death and malnutrition, although no statistical significance was shown ( $p>0.05)$. Conclusion: Patients at nutritional risk and/or malnutrition appear to be vulnerable to worse clinical outcomes.

\section{Aceito para publicação \\ 4 de novembro de 2019 \\ Submissão \\ 23 de agosto de 2019}

Endereço para correspondência

Rua Tab. Stanislau Eloy, 585 - Castelo Branco - João

Pessoa, PB, Brasil - CEP 58050-585

1. Especialista em Nutrição Clínica e Nutrição Parenteral e Enteral, Nutricionista assistencial e da Equipe Multiprofissional de Terapia Nutricional (EMTN) do Hospital Universitário Lauro Wanderley (HULW/UFPB/EBSERH), João Pessoa, PB, Brasil.

2. Especialista em Saúde Pública, Nutricionista assistencial e da Equipe Multiprofissional de Terapia Nutricional (EMTN) do Hospital Universitário Lauro Wanderley (HULW/UFPB/EBSERH), João Pessoa, PB, Brasil.

3. Especialista em Terapia Intensiva e Nutricional Parenteral e Enteral, Médica Nutróloga, coordenadora da Equipe Multiprofissional de Terapia Nutricional (EMTN) do Hospital Universitário Lauro Wanderley (HULW/UFPB/EBSERH), João Pessoa, PB, Brasil.

4. Doutora em Nutrição, Nutricionista assistencial do Hospital Universitário Lauro Wanderley (HULW/UFPB/EBSERH), João Pessoa, PB, Brasil.

5. Especialista em Terapia Intensiva e Nutricional Parenteral e Enteral, Enfermeira assistencial e enfermeira da Equipe Multiprofissional de Terapia Nutricional (EMTN) do Hospital Universitário Lauro Wanderley (HULW/UFPB/EBSERH), João Pessoa, PB, Brasil.

6. Nutricionista Residente pelo Programa de Residência Multiprofissional em Saúde Hospitalar - Ênfase em Paciente Crítico do Hospital Universitário Lauro Wanderley (HULW/UFPB/EBSERH), João Pessoa, PB, Brasil. 


\section{INTRODUÇ̃̃O}

paciente crítico é aquele que se encontra em fragilizada condição clínica, na iminência de ameaça à vida, com disfunção ou perda da funcionalidade de algum órgão, requerendo cuidados imediatos por toda equipe multiprofissional. Dentre outros insultos, esse tipo de paciente evolui com quadro de desnutrição, sarcopenia, atrofia de fibras musculares e cardíacas, ventilação mecânica e, consequentemente, maior tempo de permanência hospitalar?

Quanto ao estado nutricional desse tipo de paciente, são usados métodos de avaliação combinados, sejam objetivos e/ou subjetivos, como antropometria, marcadores bioquímicos, história nutricional pregressa, de modo a se elucidar um diagnóstico nutricional e, com isso, se planejar uma intervenção nutricional adequada ${ }^{2}$. Por meio da avaliação nutricional, é possível identificar precocemente alterações no estado nutricional do paciente e, a partir disto, determinar suas necessidades e prioridades na terapia nutricional. Porém, vale destacar que os pacientes que apresentam maior risco nutricional em uma unidade de terapia intensiva (UTI) requerem uma avaliação nutricional completa ${ }^{1,2}$. Contudo, algumas ferramentas podem ser impróprias para avaliação do paciente crítico, visto significativas mudanças de fluidos que os mesmos apresentam ${ }^{3}$.

A desnutrição, por sua vez, pode ser definida como um estado resultante da deficiência de nutrientes, que pode causar alterações na composição corporal, funcionalidade e estado mental, e que pode levar a um desfecho clínico negativo ${ }^{4}$. Em clássicos estudos nessa área, foi visto que, em período de quase 20 anos, a prevalência de desnutrição apresentou tendência crescente. Trata-se de um inquérito brasileiro, Inquérito Brasileiro de Avaliação Nutricional Hospitalar (IBRANUTRI) ${ }^{5}$ que, em 1998, levantou 4 mil pacientes, apontando que $48,1 \%$ estavam desnutridos. Revisão sistemática elaborada por Correia et al. ${ }^{6}$ demonstrou a manutenção dessa alta prevalência.

Uma oferta adequada de nutrientes é essencial para prevenir perdas, manter o equilíbrio imunológico e auxiliar na diminuição das complicações metabólicas, consequentemente mais acelerada é a recuperação e menor o tempo de permanência hospitalar ${ }^{7}$. Em estudo sobre terapia nutricional e desfecho clínico, Franzosi et al. ${ }^{8}$ mencionam que o objetivo da terapia nutricional em UTI inclui o fornecimento de aporte nutricional adequado, ao passo que previne deficiências nutricionais, atenuando, assim, a perda de massa muscular magra, evitando complicações e, por fim, otimizando desfecho clínico.

Diante do exposto, esse estudo tem como objetivo avaliar o estado nutricional e desfechos clínicos em pacientes críticos internados em hospital universitário.

\section{MÉTODO}

Estudo transversal realizado na unidade de terapia intensiva do Hospital Universitário Lauro Wanderley (HULW/ UFPB/EBSERH, João Pessoa-PB), entre os meses de março e dezembro de 2018. A amostra foi composta por todos os pacientes adultos entre 18 e 99 anos, de ambos os sexos, cujo prontuário possuísse o formulário de acompanhamento nutricional padronizado pelo serviço de nutrição do hospital, devidamente preenchido, com tempo de permanência na UTI igual ou superior a 72 horas e que fizeram uso da terapia nutricional enteral (TNE) por sondas ou ostomias. Como critérios de exclusão definiu-se evolução para alta ou óbito dentro das primeiras 72 horas e o não uso da TNE.

A terapia nutricional da referida instituição é realizada diariamente e de forma ininterrupta, conforme quadro clínico e necessidades nutricionais de cada paciente. Todos os pacientes submetidos à TNE recebem fórmulas enterais industrializadas em sistema aberto (prontas para uso) ou sistema fechado, manipuladas em sondário conforme legislação específica, com administração contínua (24 horas) controlada por bomba de infusão ou gravitacional. A dieta enteral tem como característica ser normocalórica e hiperproteica (isenta de lactose, sacarose e glúten), acrescida de módulos calóricos e/ou proteicos, conforme necessidades estimadas. No caso de pacientes que possuem necessidades metabólicas específicas, utiliza-se dieta enteral adequada à sua condição clínica.

A coleta de dados ocorreu do período entre a admissão até o momento de descontinuação da TNE, óbito ou alta da unidade. Para coleta de dados, utilizou-se formulário previamente estruturado utilizado na rotina diária do nutricionista, onde são registradas informações sobre dados de identificação (idade, gênero), dados clínicos (diagnóstico, motivo de internação, tempo de internação, desfecho), dados antropométricos (peso, altura, circunferência do braço e altura do joelho), necessidades nutricionais e variáveis relacionadas à nutrição enteral (dieta, via de aceso, administração, complicações gastrointestinais), bem como indicadores de qualidade da terapia nutricional (jejum inadequado, atendimento de meta energética em $72 \mathrm{~h}$, volume prescrito versus infundido, diarreia e constipação).

Os dados foram digitados no programa Excel para Windows ${ }^{\circledR}$. As análises foram realizadas no Programa Statistical Package for Social Sciences (SPSS) versão 13.0. As variáveis contínuas foram testadas quanto à normalidade pelo teste de Kolmogorov Smirnov. Os dados foram descritos utilizando frequências, medidas de tendência central e de variação, para variáveis normais, média e desvio padrão (DP), e não-normais, mediana e intervalo interquartílico (IQ). Para as variáveis com distribuição normal, utilizou-se o teste † de Student para comparar dois grupos independentes. Para as 
variáveis com distribuição não-normal, utilizou-se o teste de Mann Whitney para comparar dois grupos. Na comparação entre as proporções, utilizou-se o teste do qui quadrado de Pearson ou com correção de Yates para tabelas $2 \times 2$. O nível de significância adotado foi de $p<0,05$.

O projeto foi aprovado pelo Comitê de Ética em Pesquisa em Seres Humanos do Hospital Universitário Lauro Wanderley (CEP-HULW), CAAE n ${ }^{\circ}$ 131924.19.0.0000.5183, de acordo com a Resolução n 196/96 do Conselho Nacional de Saúde.

\section{RESULTADOS}

A amostra foi composta por 116 pacientes, de ambos os sexos, sendo a maioria do sexo feminino $(53,4 \%, n=62)$, com idade média de 46 anos (IQ $31-53$ anos) e faixa etária prevalente a de adulto meia-idade (54,5\%; entre 40 e 60 anos). A principal via de acesso para o suporte nutricional foi a via oral $(55,6 \%)$, seguida da enteral $(30,6 \%)$. Dentre os pacientes analisados, houve $79,1 \%$ de altas, como desfecho clínico. Em relação ao risco nutricional avaliado pelo Nutric escore, $24(61,5 \%)$ pacientes apresentaram escore baixo e $15(38,5 \%)$, alto.

A Tabela 1 descreve a comparação de média e desvio padrão dos indicadores do estado nutricional entre os pacientes em TNE. Observa-se que o sexo masculino apresentou maior média ou mediana de idade, peso, altura e Nutric escore, quando comparado ao sexo feminino, porém com diferenças estatisticamente apenas em relação à altura $(p<0,05)$. O sexo feminino apresentou maior média de IMC e circunferência braquial (CB), sem diferenças estatisticamente significante.
Tabela 1 - Média e desvio padrão dos indicadores do estado nutricional de pacientes em uso de terapia nutricional enteral em unidade de terapia intensiva de um hospital universitário, 2019.

\begin{tabular}{|c|c|c|c|c|}
\hline \multirow[t]{2}{*}{ Variáveis } & Total & $\begin{array}{c}\text { Sexo } \\
\text { Masculino }\end{array}$ & $\begin{array}{c}\text { Sexo } \\
\text { Feminino }\end{array}$ & \multirow[t]{2}{*}{$\begin{array}{l}\text { Valor } \\
\text { de } p\end{array}$} \\
\hline & Média ( \pm DP) & Média ( \pm DP) & Média ( \pm DP) & \\
\hline Idade (anos) ${ }^{*}$ & $46(31-53)$ & $47(35-52,5)$ & $39(29,5-53,5)$ & $0,445^{\mathrm{a}}$ \\
\hline Pes & $\begin{array}{c}64,8 \\
(54,7-74)\end{array}$ & $\begin{array}{c}69 \\
(57,2-76,5)\end{array}$ & $\begin{array}{c}61 \\
(53,4-71,8)\end{array}$ & $0,084^{a}$ \\
\hline Altura (m) & $\begin{array}{c}1,64 \\
( \pm 0,09)\end{array}$ & $\begin{array}{c}1,69 \\
( \pm 0,08)\end{array}$ & $\begin{array}{c}1,60 \\
( \pm 0,07)\end{array}$ & $<0,05^{b}$ \\
\hline IMC $\left(\mathrm{kg} / \mathrm{m}^{2}\right)^{\star}$ & $\begin{array}{c}23,25 \\
(19,8-27,8)\end{array}$ & $\begin{array}{c}23,14 \\
(20,0-27,6)\end{array}$ & $\begin{array}{c}23,3(19,7- \\
28,0)\end{array}$ & $0,897^{a}$ \\
\hline $\mathrm{CB}$ & $24,93( \pm 4,3)$ & $23,96( \pm 3,88)$ & $25,65( \pm 4,63)$ & $0,244^{b}$ \\
\hline Nutric escore & $3,77( \pm 2,2)$ & $4,0( \pm 2,26)$ & $3,55( \pm 2,23)$ & $0,536^{b}$ \\
\hline
\end{tabular}

a Teste t de Mann Whitney: variáveis não normais descritas como mediana e intervalos interquartílicos;

b Teste t de Student: variáveis normais descritas como média e desvio padrão (DP).

$\mathrm{CB}=$ circunferência braquial; $\mathrm{IMC}$ = índice de massa corporal.

Em relação ao diagnóstico nutricional, foi identificada frequência importante de desnutrição segundo o indicador CB (73\%), entretanto, pelo IMC, encontrou-se menor frequência de déficit nutricional $(17,4 \%)$, contudo, a maioria dos pacientes apresentou risco nutricional baixo $(61,5 \%$, $\mathrm{n}=24$ ) (Figura 1).

Pacientes desnutridos pelos indicadores IMC, $C B$ e os que apresentaram risco nutricional pelo Nutric escore obtiveram maior mediana de tempo de internamento quando comparado aos sem déficits nutricionais ou sem risco nutricional (IMC: 10 dias IQ 4,3-20,5 versus 7,5 dias IQ 4 -12,5, $p=0,209 ; C B: 8$ dias IQ 5 - 19 versus 7 dias IQ $6-11,8$, $p=0,603$; Nutric escore: 18 dias IQ $8-23$ versus 8,5 dias

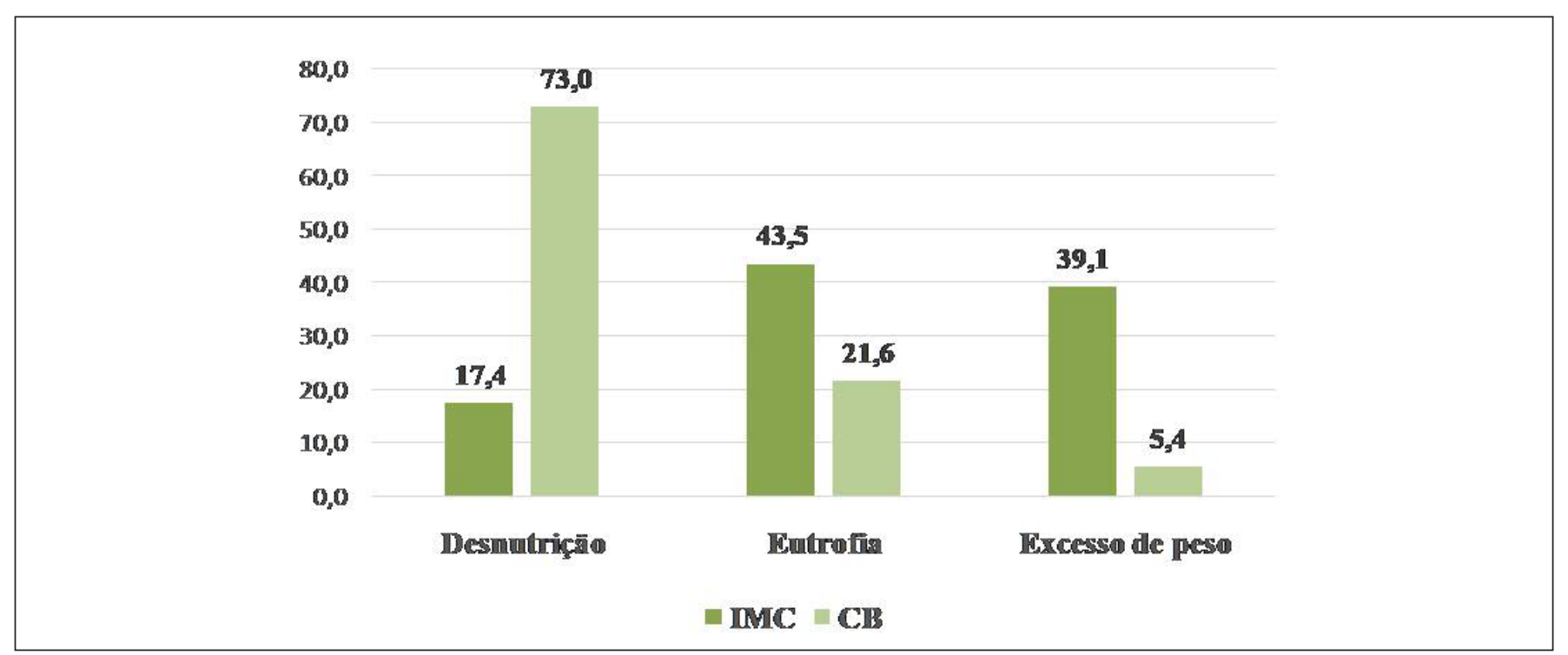

Figura 1 - Estado nutricional segundo IMC e CB de pacientes em uso de terapia nutricional enteral em unidade de terapia intensiva de um hospital universitário, 2019. $C B=$ circunferência braquial; IMC = indice de massa corporal. 
IQ 9 - 19,8, $p=0,212$; respectivamente), contudo, sem diferenças estatisticamente significantes.

A associação entre desfecho clínico e estado nutricional segundo diferentes métodos foi descrita na Tabela 2. Os dados apontam que, entre os pacientes que foram a óbito, houve maior frequência de desnutrição aferida pela CB e de risco nutricional avaliado pelo Nutric escore, quando comparados aos que tiveram alta da UTI $(88,9 \%$ versus $66,7 \%$, $p=0,408 ; 44,4 \%$ versus $36,7 \%, p=0,976$, respectivamente), contudo, sem diferenças estatisticamente significantes.

Tabela 2 - Associação entre desfecho clínico e estado nutricional segundo diferentes métodos em pacientes em uso de terapia nutricional enteral em unidade de terapia intensiva de um hospital universitário, 2019.

\begin{tabular}{lccc}
\hline Variável & \multicolumn{2}{c}{ Desfecho } & Valor de $\mathbf{p}$ \\
\cline { 2 - 3 } & $\begin{array}{c}\text { Alta } \\
\mathrm{n}(\%)\end{array}$ & $\begin{array}{c}\text { Óbito } \\
\mathrm{n}(\%)\end{array}$ & \\
\hline Estado nutricional (IMC) & & & $0,1731^{\mathrm{a}}$ \\
Desnutrido & $15(20)$ & $1(6,3)$ & \\
Eutrófico & $29(38,7)$ & $10(62,5)$ & \\
Sobrepeso/Obesidade & $31(41,3)$ & $5(31,3)$ & \\
\hline Estado nutricional (CB) & & & $0,408^{\mathrm{a}}$ \\
Desnutrido & $18(66,7)$ & $8(88,9)$ & \\
Eutrófico & $7(25,9)$ & $1(11,1)$ & \\
Sobrepeso/Obesidade & $2(7,4)$ & - & $0,976^{\mathrm{b}}$ \\
\hline Nutric Escore & & & \\
Baixo & $19(63,3)$ & $5(55,6)$ & \\
Alto & $11(36,7)$ & $4(44,4)$ & \\
\hline
\end{tabular}

${ }^{a}$ Teste de Pearson; ' ${ }^{b}$ Correção de Yates.

$\mathrm{CB}=$ circunferência braquial; $\mathrm{IMC}$ = índice de massa corporal.

\section{DISCUSSÃO}

Nos últimos anos, houve aumento das taxas de sobrevida de pacientes com doença crítica e, consequentemente, do número de morbidades deles em decorrência da permanência prolongada nas UTIs. Devido a esta longa permanência associada ao estado hipermetabólico, os pacientes frequentemente evoluem com desnutrição, podendo acarretar o óbito?.

O IBRANUTRI demonstrou que a prevalência de desnutrição nos pacientes hospitalizados era de $48,1 \%$, sendo que $12,6 \%$ apresentavam desnutrição grave ${ }^{5}$. Um estudo latino-americano com 1053 pacientes internados em 116 unidades de terapia intensiva, incluindo o Brasil, identificou $74,1 \%$ de pacientes com desnutrição moderada ou grave ${ }^{6}$.

No presente trabalho, observou-se maior frequência do gênero feminino, com média de idade de 46 anos. Um estudo realizado em 15 UTIs de hospitais em Portugal, diferentemente da presente casuística, obteve média de 64 anos, sendo $64,8 \%$ homens $^{10}$. Estudo realizado no Brasil, na cidade de Anapólis -Goiás, demonstrou também a prevalência do sexo masculino nas internações e idade média à internação de 59 anos $^{11}$.

O presente estudo constatou que o tempo médio de permanência para pacientes em risco de desnutrição, avaliado pelo Nutric Score, foi significativamente maior (10,5 dias) do que o tempo médio de permanência para pacientes que não apresentavam esse risco (4 dias); esse tempo de internação também foi maior, segundo os indicadores IMC e $\mathrm{CB}$, respectivamente. Resultado semelhante foi demonstrado em outro estudo, no qual o tempo de internação hospitalar foi maior nos pacientes desnutridos, os quais permaneceram no hospital por 16,7 $\pm 24,5$ dias (mediana de 9 dias) versus $10,1 \pm 11,7$ dias nos nutridos (mediana de 6 dias) ${ }^{12}$. Outro estudo demonstrou que o tempo médio de internação total do hospital dos desnutridos graves foi de 8 (IQ 5-16) dias, o tempo total mediano total da UTI foi de $3(1-7)$ dias e a taxa geral de mortalidade foi de $16,64 \%^{13}$. Caracterizando, assim, que pacientes desnutridos tendem a apresentar maior tempo de permanência hospitalar, conforme demonstra o estudo.

Quanto ao desfecho clínico do presente estudo, percebeuse que $79,1 \%$ dos pacientes receberam alta e $20,9 \%$ foram a óbito, o que corrobora com o estudo realizado na UTI Geral e Cardiológica do Hospital Universitário da Universidade Federal do Maranhão ${ }^{14}$. Em tal estudo, $54,7 \%$ dos pacientes obtiveram alta e $45,3 \%$ foram a óbito ${ }^{14}$.

A porcentagem de pacientes da amostra deste trabalho classificados em risco nutricional alto foi de $38,5 \%$ pelo instrumento de triagem Nutric Score, divergindo de outros estudos em que a porcentagem se manteve entre $48,6 \%$ e $56,9 \%^{10,15,16}$, enquanto convergiu com os achados de outro estudo em hospitais de Portugal com 1152 doentes, no qual a frequência de risco nutricional variou entre 28,5\% e 47,3\%17.

Quanto ao estado nutricional dos pacientes deste estudo, foi identificada frequência de desnutrição, avaliada por meio da $C B$, quatro vezes maior do que a obtida pelo IMC. Essa diferença entre os métodos também foi encontrada no estudo de Stefanello \& Poll ${ }^{18}$ e Martins et al. ${ }^{19}$, que observaram que a $C B$ diagnosticou mais que o dobro de desnutrição, em relação ao IMC.

Esses resultados demonstram que o IMC é um simples indicador de estado nutricional e, como outros parâmetros de avaliação, tem suas limitações, especialmente no paciente crítico. Demonstram, ainda, maior sensibilidade da CB em identificar a desnutrição dos pacientes críticos e reforçam a importância da utilização de vários métodos para a definição de um diagnóstico nutricional mais preciso, visto que, nos pacientes internados em UTI, há muitas limitações ao utilizar 
um método específico. Isso porque esses pacientes podem apresentar alterações nos fluidos corporais, bem como dificuldade de mensuração e obtenção de dados confiáveis. Apesar da relevância da avaliação nutricional no paciente crítico, ainda não existe, na literatura, um padrão-ouro para avaliação nutricional desses pacientes ${ }^{3}$.

O diagnóstico da desnutrição tem o potencial de identificar comorbidades modificáveis e/ou esclarecer o prognóstico. É uma condição comum que resulta de um desequilíbrio nutricional e/ou inflamação sistêmica ${ }^{13}$. A desnutrição pode levar a alterações no metabolismo, com piora no processo de cicatrização, piora do estado imunológico, aumentando, assim, a taxa de infecções e complicações ${ }^{20}$. Essas complicações podem influenciar os resultados clínicos, levar a aumento de morbidade, mortalidade, tempo de internação, reinternação e custos ${ }^{9,21,22}$. Muitas vezes, os pacientes entram no hospital desnutridos ou em risco de desnutrição e experimentam declínio nutricional durante a estadia, colocando-os em maior risco de resultados adversos após a alta hospitalar ${ }^{23}$. Em hospitais de todo o mundo, verifica-se que a desnutrição influencia muito as doenças e a recuperação de doenças e, portanto, é um grande desafio na área de saúde ${ }^{21}$.

Uma recente revisão sistemática relatou que a desnutrição pode estar presente entre 38\% a $78 \%$ dos pacientes críticos internados e, ainda, que essa desnutrição progride à medida que o tempo passa, contribuindo para aumento da morbimortalidade ${ }^{23}$.

Os dados do presente estudo apontam que, entre os pacientes que foram a óbito, houve maior frequência de desnutrição aferida pela $\mathrm{CB}$ e de risco nutricional avaliado pelo Nutric escore, quando comparados aos que tiveram alta da UTI, apesar de não apresentarem diferenças estatísticas. Um estudo semelhante também apontou mortalidade hospitalar significativamente influenciada pelo estado nutricional dos pacientes, no qual, mortalidade nos pacientes desnutridos foi de $12,4 \%$ versus $4,7 \%$ nos bem nutridos ( $R R=2,63)^{12}$. Hiura et al. ${ }^{13}$ mostraram em estudo recente que $12,95 \%$ da sua amostra foi diagnosticada com desnutrição grave, dos quais 231 (31,82\%) morreram no hospital. Neste interim, pode-se considerar que a desnutrição afeta negativamente no desfecho clínico do paciente.

Algumas limitações metodológicas merecem ser mencionadas, entre elas o fato de o estudo ter sido conduzido em uma única UTI, e, assim, os resultados não serem aplicáveis a outras instituições. Além disso, a dificuldade de se aplicar outros instrumentos de avaliação nutricional.

\section{CONCLUSÃO}

Diante do exposto, observou-se que toda dinâmica, desde a admissão do paciente, triagem, avaliação, delineamento da terapia nutricional e acompanhamento, deve ser rigorosamente trabalhada, visando, sobretudo, a prevenção e/ou tratamento da desnutrição, sendo esse um fator que pode determinar o desfecho clínico do paciente.

Outrossim, as ferramentas de avaliação para esse tipo de paciente são limitadas, assim, não se pode garantir acurácia do estado nutricional, apesar de ser usado na maioria dos hospitais, devido à escassez de recursos mais fidedignos.

Por outro lado, todo este contexto se torna paradoxal, quando partimos da premissa de que, quanto mais o paciente é desnutrido, mais retardada será sua recuperação e maiores serão os custos hospitalares. Tratar esse tipo de paciente com protocolos que integrem uma abordagem interdisciplinar, com início precoce da terapia nutricional e salvaguardando os aspectos do cuidado geral do paciente crítico desnutrido, parece ser um bom caminho para diminuir a incidência de desnutrição e os desfechos clínicos a ela associados.

\section{REFERÊNCIAS}

1. Toledo DO, Piovacari SMF, Horie LM, Matos LBN, Castro MG, Ceniccola GD, et al. Campanha "Diga não à desnutrição": 11 passos importantes para combater a desnutrição hospitalar. BRASPEN J. 2018;33(1):86-100.

2. Beserra EA, Rodrigues PA, Lisboa AQ. Validação de métodos subjetivos para estimativa do índice de massa corporal em pacientes acamados. Com Ciências Saúde. 2011;22(1):19-26.

3. Santos HVD, Araújo IS. Impacto do aporte proteico e do estado nutricional no desfecho clínico de pacientes críticos. Rev Bras Ter Intensiva. 2019;31(2):210-6.

4. Anthony PS. Nutrition screening tools for hospitalized patients. Nutr Clin Pract. 2008;23(4):373-82.

5. Correia MITD, Caiaffa WT, Waitzberg DL. Inquérito brasileiro de avaliação nutricional hospitalar (IBRANUTRI): metodologia do estudo multicêntrico. Rev Bras Nutr Clín. 1998;13(1):30-40.

6. Correia MITD, Perman MI, Waitzberg DL. Hospital malnutrition in Latin America: a systematic review. Clin Nutr. 2017;36(4):958-67.

7. Paz LSC, Couto AV. Avaliação nutricional em pacientes críticos: revisão de literatura. BRASPEN J. 2016;31(3):269-77.

8. Franzosi OS, Abrahão CLO, Loss SH. Aporte nutricional e desfechos em pacientes críticos no final da primeira semana na unidade de terapia intensiva. Rev Bras Ter Intensiva. 2012;24(3):263-9.

9. Silva IR, Estumano NV, Nascimento PMS, Miranda TV, Araújo MS, Santos CH. Alterações do estado nutricional em pacientes recebendo terapia nutricional em um hospital de referência em Belém-PA. BRASPEN J. 2018;33(4):428-34.

10. Heyland DK, Dhaliwal R, Wang M, Day AG. The prevalence of iatrogenic underfeeding in the nutritionally 'at-risk' critically ill patient: results of an international, multicenter, prospective study. Clin Nutr. 2015;34(4):659-66.

11. Castro RB, Barbosa NB,Alves T, Najberg E. Perfil das internações em unidades de terapia intensiva adulto na cidade de Anapólis Goiás - 2012. Rev Gest Sist Saúde. 2016;5(2):115-24.

12. Correia MI, Waitzberg DL. The impact of malnutrition on morbidity, mortality, length of hospital stay and costs evaluated through a multivariate model analysis. Clin Nutr. 2003;22(3):235-9.

13. Hiura G, Lebwohl B, Seres DS. Malnutrition diagnosis in critically ill patients using 2012 Academy of Nutrition and Dietetics/American Society for Parenteral and Enteral Nutrition standardized diagnostic characteristics is associated with 
longer hospital and intensive care unit length of stay and increased in-hospital mortality. JPEN J Parenter Enteral Nutr. 2019. doi: 10.1002/jpen.1599.

14. Rocha AJSC, Oliveira ATV, Cabral NAL, Gomes RS, Guimarães TA, Rodrigues WB, et al. Causas de interrupção de nutrição enteral em unidades de terapia intensiva. Rev Pesq Saúde. 2017;18(1):49-53.

15. Mendes R, Policarpo S, Fortuna P, Alves M, Virella D, Heyland DK; Portuguese NUTRIC Study Group. Nutritional risk assessment and cultural validation of the modified NUTRIC score in critically ill patients: a multicenter prospective cohort study. J Crit Care. 2017;37:45-9.

16. Coltman A, Peterson S, Roehl K, Roosevelt H, Sowa D. Use of 3 tools to assess nutrition risk in the intensive care unit. JPEN J Parenter Enteral Nutr. 2015;39(1):28-33.

17. Matos L, Teixeira MA, Henriques A, Tavares MM, Alvares L, Antunes A, et al. Registro do estado nutricional em anotações de pacientes hospitalizados. Acta Med Port. 2007;20(6):503-10.
18. Stefanello MD, Poll FA. Estado nutricional e dieta enteral prescrita e recebida por pacientes de uma unidade de terapia intensiva. ABCS Health Sci. 2014;39(2):71-6.

19. Martins RC, Vital WC, Amaral JF, Volp AC. Perfil nutricional de pacientes internados em unidade de terapia intensiva. Nutr Clín Diet Hosp. 2017;37(4):40-7.

20. Zheng H, Huang Y, Shi Y, Chen W, Yu J, Wang X. Nutrition status, nutrition support therapy, and food intake are related to prolonged hospital stays in China: results from the NutritionDay 2015 survey. Ann Nutr Metab. 2016;69(3-4:215-25.

21. Allison SP. Desnutrição, doença e resultado. Nutrition. 2000;16:590-1.

22. SauerAC, Goates S, MaloneA, Mogensen KM, Gewirtz G, Sulz I, et al. Prevalence of malnutrition risk and the impact of nutrition risk on hospital outcomes: results from NutritionDay in the U.S. JPEN J Parenter Enteral Nutr. 2019;43(7):918-26.

23. Lew CCH, Yandell R, Fraser RJL, Chua AP, Chong MFF, Miller M. Association between malnutrition and clinical outcomes in the intensive care unit: a systematic review. JPEN J Parenter Enteral Nutr. 2017;41(5):744-58.

Local de realização do estudo: Hospital Universitário Lauro Wanderley, João Pessoa, PB, Brasil.

Conflito de interesse: As autoras declaram não haver.

Foi apresentado como trabalho de destaque no XXIII Congresso Brasileiro de Nutrição Parenteral e Enteral, entre 20 a 23 de outubro de 2019, em Foz do Iguaçu-PR. 